\title{
USE OF PARTICLE SWARM OPTIMIZATION ALGORITHM FOR DIGITALIZED SINEWAVE SIGNAL PARAMETERS ESTIMATION
}

\author{
Pierfrancesco Raimondo \\ Dept. of Informatics, Modelling, Electronics and System Eng., University of Calabria, \\ 87036 Rende-CS, Italy, p.raimondo@dimes.unical.it
}

\begin{abstract}
In the paper is proposed a procedure based on the particle swarm optimization algorithm for parameters estimation of sinewave signals as: amplitude, phase, frequency and offset. Differently from the classical method used to solve this problem (the sine-fitting algorithms), the proposed procedure considers the estimation problem as an optimization one. In fact, the particle swarm algorithm tends to global solution instead of a local solution. The proposed procedure preliminarily estimates the raw value of the parameters under investigation by a time analysis of the input signal. Successively, these values are used by the particle swarm algorithm for the final estimation result. The tests of the proposed procedure determine the most effective cost function for the algorithm and confirm that the achievable performances are in according with the sine fitting algorithm. Moreover, the execution time for the proposed procedure is lower than the sine fitting, making it an interesting alternative. Copyright $(\mathcal{C}$ Research Institute for Intelligent Computer Systems, 2017. All rights reserved.
\end{abstract}

Keywords: Particle swarm application, sine fitting, optimization technique.

\section{INTRODUCTION}

The estimation of sinusoidal signal parameters as amplitude, phase, frequency and offset, is a crucial problem in several application fields [1]-[3]. The main solution proposed in literature are based on the sine fitting algorithms [4]-[8], multi-sine fitting algorithm that is its multi harmonic evolution, [9][11], and Discrete Fourier Transform (DFT) [12].The first two solutions have the problems that their accuracy depends on the number of samples and signal period analyzed. Moreover, they require to accurately preliminary evaluate the frequency of the signal, in order to not converge to a local minimum of the solution or to diverge. Instead, the DFT requires to analyze a finite number of the signal period, in order to provide a result not affected by spectral leakage. Moreover, its frequency resolution depends on the ratio between the sampling frequency and the number of samples analyzed.

In the paper is analyzed the problem of the estimation of the sinusoidal signal parameters as an optimization problem and it is solved by using the Particle Swarm optimization Algorithm (PSA). The PSA is an heuristic algorithm [13], [14], developed by the natural observation of the swarm or insect colonies [15]. Each agent or particle of the swarm works without a central control but adjust its behavior versus the perception of the other agents in its neighborhood. In this way, moving in the solution space of a problem, the PSA searches the global optimum of an objective function. By considering the solution space constituted by the unknown parameters of the sinusoidal signal and as objective function the best fit parameters for the acquired samples the PSA can be used instead the sine fitting algorithms. Numerical tests are executed to determine the accuracy in the parameters estimation of the PSA in different scenarios. Moreover, it is introduced a procedure to set the PSA parameters to reduce the execution time and increase the result accuracy. The achieved accuracy is compared with the sine fitting algorithm one that is the golden standard for this estimation.

The paper is organized as follows: preliminary the basis of the particle swarm algorithm is introduced for sake of completeness. Successively, the procedure based on PSA to solve the parameters estimation problem is described. Following, the proposed procedure is characterized in order to verify the influence of different operating conditions on the estimation results. Moreover, the results achieved are compared with the sine fitting algorithm results. Finally, the conclusions are drawn.

\section{BASIS OF PARTICLE SWARM ALGORITHM}

The PSA is a heuristic methodology developed to search the solution of an objective optimization problem [15]-[20]. It is based on a population of agents or particles that sharing information. Each 
particle, in a specific time instant, is characterized by a position in the space solution. In order to converge versus the best solution, the position of the particles is iteratively updated, and moved from the previous position with a velocity vector obtained as a combination of the results of the other particles. Then in each iteration, the particles can change the search direction converging to the optimal solution of the problem. The convergence criteria are detailed in [21].

To determine at the iteration $\mathrm{i}+1$ the velocity vector $V_{i+1}$ and position $X_{i+1}$ of the particle $p$ the following equations are used:

$$
\begin{aligned}
& \boldsymbol{V}_{i+1, p}=\boldsymbol{\omega}_{i} * \boldsymbol{V}_{i, p}+\boldsymbol{c}_{1} * \operatorname{rand}\left(0 *\left(\boldsymbol{P B} \boldsymbol{B}_{p}-\boldsymbol{X}_{i, p}\right)+\boldsymbol{c}_{2} * \operatorname{rand} 0 *\left(\boldsymbol{S B}-\boldsymbol{X}_{i, p}\right)\right. \\
& \boldsymbol{X}_{i+1, p}=\boldsymbol{X}_{i, p}+\boldsymbol{V}_{i+1, p}
\end{aligned}
$$

where $c_{1}$ and $c_{2}$ are positive constants that quantify the strength of the particle attraction, $\omega_{i}$ is the particles inertia, $\boldsymbol{r a n d}()$ is a function that generates a random vector with values within the range $(0,1)$, that permits the exploration of the space, $\boldsymbol{P} \boldsymbol{B}_{p}$ is the best position for the particle $p$ and $\boldsymbol{S B}$ is the best position for the whole swarm. The term in (1) related to $\mathrm{c}_{1}$ is called cognitive component because is related to the perception of each particle about the optimal solution, instead the term related to $c_{2}$ is called social component and represents the information exchanged by the swarm agents.

The inertial is updated in each iteration by oscillating in predefined inertia range.

The complete algorithm of PSA is described in Fig. 1. The main steps are:

1. Initialize the swarm parameters: the algorithm sets the maximum number of iteration that can be executed, the number of particle composing the swarm, set an initial random velocity vector. The particles are distributed uniformly in solution space. Moreover, are set the bound for the solution (in order to limit the solution space) and for the inertia coefficients. Once the initialization is completed the algorithm evaluate the objective function to be minimized for all the particles. $\boldsymbol{P} \boldsymbol{B}_{p}$ is set as the current solution for the particle $p$. Instead, $\boldsymbol{S B}$ is set as the best solution among all the solution achieved.

2. Swarm updating: the velocity and the position of each particle is update by using the (1). Preliminary is checked if the velocity and position are bound in the value defined in the initialization step. If it is true, it is checked if the new position is better than the previous one for the objective function. In this case $\boldsymbol{P} \boldsymbol{B}_{p}$ value is updated. Once the updating is completed the best solution among the solution of the swarm is determined and is used to set $\boldsymbol{S B}$.

3. Stopping criterion check: the algorithm repeats the step 2 until it reaches a stopping criteria composed by different checks. In particular, is verified if the algorithm has reached the imposed maximum iteration or the best results for the objective function has no change for a preestablished number of times $T_{M a x}$.

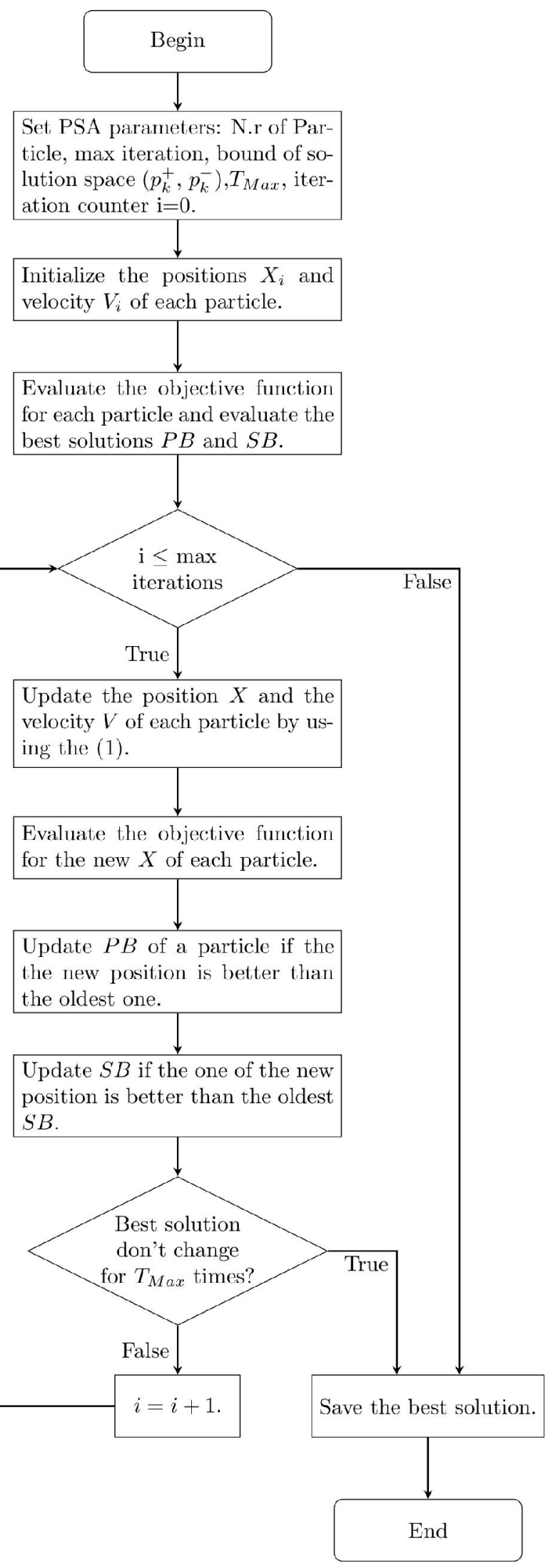

Fig. 1 - Computation flowchart of Particle Swarm Algorithm. 


\section{PROCEDURE FOR THE SINUSOIDAL SIGNAL PARAMETERS ESTIMATION BASE ON PSA}

The model of the signal $y(t)$ considered is the following:

$$
y(t)=S \sin [\omega t+\phi]+C+n(t)
$$

where $S, C$ and $\phi$ are the unknown amplitude, offset and phase of the sinusoidal signal, respectively, $\omega=2 \pi f$ is the unknown angular frequency, with $f$ the signal frequency and $n(t)$ is noise superimposed to the signal. The unknown parameters are the position $\boldsymbol{X}$ of the swarm particles:

$$
\boldsymbol{X}=\left[\begin{array}{llll}
S, & \phi, & \omega, & C
\end{array}\right]
$$

In order to apply the PSO is necessary to preliminary define the bound of the solution space for $\boldsymbol{X}$ in which the objective function will be minimized. For this reason, the proposed procedure is divided in two successive steps. In the preliminary step the bound of the solution space is determined by the time analysis of the input signal. Successively, in the second step the PSA is applied to the input signal in the determined solution space to obtain the minimization of the objective function.

\subsection{PARTICLE BOUND AND OBJECTIVE FUNCTION SELECTION}

In order to restrict the solution space for $\boldsymbol{X}$ a preliminary procedure is proposed. The procedure analyses the positive $p k^{+}=\left[p k_{1}^{+}, p k_{2}^{+}, \ldots, p k_{M}^{+}\right]$and negative $p k^{-}=\left[p k_{1}^{-}, p k_{2}^{-}, \ldots, p k_{M}^{-}\right]$peaks of the acquired signal. In fact, as can be seen in Fig. 2, the bound for $S$ is selected as the half of the mean value of the difference between $p k^{+}$and $p k^{-} \pm$three times of the standard deviation of the difference. The bound of the space solution for $C$ is set as the mean value of the sum of $p k^{+}$and $p k^{-} \pm$three times of the standard deviation of the sum. The bound for the frequency is determined by the peaks distance. In fact, each peak corresponds to the beginning of a period then the inverse of the time distance between a peak and the successive one can be used as frequency estimation. The bound for $\omega$ is set as the mean value \pm three times of the standard deviation of the inverse of the time distance between the peaks. Finally, to provide a raw estimation of the phase is considered the time interval $\Delta t$ between the beginning of the acquisition and the first peak, and the raw value of the frequency. With these two parameters, the raw value of $\phi$ can be estimated as:

$$
\phi=\frac{\pi}{2}-\Delta t \omega
$$

In this case the bound is set as the $10 \%$ of the estimated $\Delta t$.

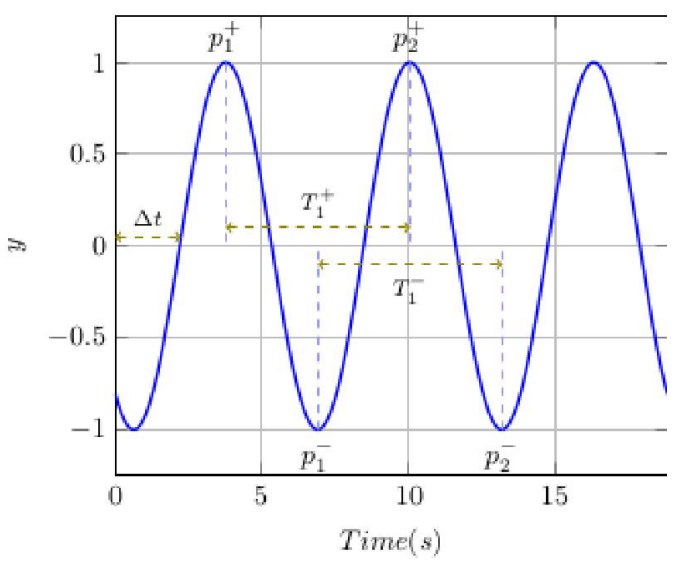

Fig. 2 - PSA preliminary evaluation of swarm position exemplification.

In order to select the objective function several options are compared, considering as criterion for choosing the error in frequency estimation.

The objective function considered are:

1. Integral of the error:

$$
f i t_{1}(\boldsymbol{X})=\frac{1}{N} \sum_{i=1}^{N}\left(y_{i}-y_{i}^{\prime}\right)
$$

2. Integral of the absolute error:

$$
f i t_{2}(\boldsymbol{X})=\frac{1}{N} \sum_{i=1}^{N}\left|\left(y_{i}-y_{i}^{\prime}\right)\right|
$$

3. Integral of the square error:

$$
f i t_{3}(\boldsymbol{X})=\frac{1}{N} \sum_{i=1}^{N}\left(y_{i}-y_{i}^{\prime}\right)^{2}
$$

where $\mathrm{N}$ is the number of samples and $\mathrm{y}^{\prime}$ is the signal reconstructed with the parameters of the particle.

Fig. 3 shows the mean error in the frequency estimation for the three objective functions versus the number of samples processed. The test is repeated 100 times for each value of number of samples and the signal is generated with white Gaussian noise superimposed. The Signal to Noise Ratio is equal to $30 \mathrm{~dB}$. The figure shows as fit $t_{l}$ has an error higher then both the other objective functions. Instead, $\mathrm{fit}_{2}$ and $\mathrm{fit}_{3}$ has about the same error, but fit $_{2}$ requires lower number of operation to be evaluated then is chosen as objective function. 


\subsection{EXECUTION TIME}

In order to evaluate the computational complexity versus the number of processed samples, the normalized execution time is taken into consideration. The normalized execution time is a performance index not influenced by the specific hardware and it is defined as the ratio of the execution time of each test to the highest processing time of the complete procedure [22]. In order to demonstrate the advantages of the proposed method the normalized execution time is compared with the same index obtained by using the 4-parameter sine fitting. The method and the convergence criteria for the 4-parameter sine fitting are described in [23].

In the tests are considered a sinusoidal signal with frequency $49.7 \mathrm{~Hz}$, amplitude $1 \mathrm{~V}$, offset $0.1 \mathrm{~V}$, random phase and with a superimposed noise. The signal to noise ratio in the tests are imposed equal to $50 \mathrm{~dB}$. For each number of processed samples tested 100 evaluations are repeated and the mean value of the execution time is considered.

Fig. 4 shows the result of the test. The figure shows as both the method increasing the normalized execution time with the number of samples, but the sine fitting algorithm is a magnitude of order higher than the PSA result.

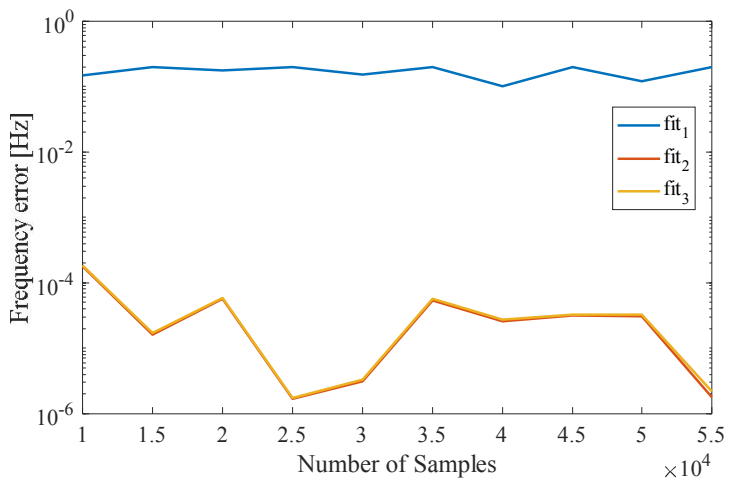

Fig. 3 - Objective functions comparison in the frequency estimation for noisy signal.

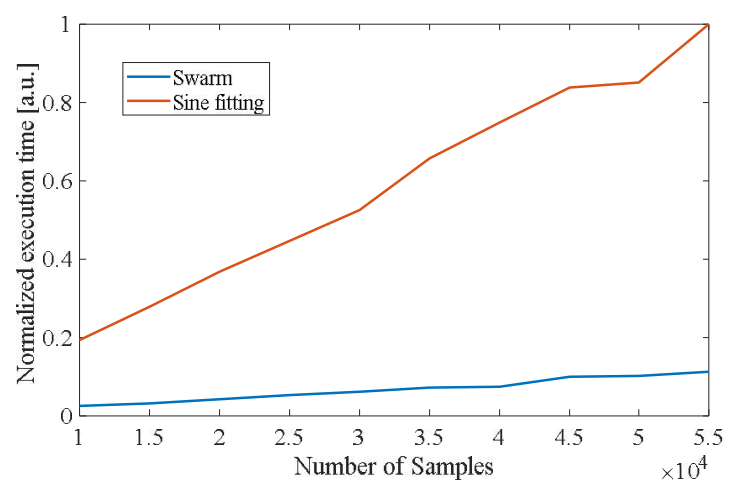

Fig. 4 - Comparison of the normalized execution time for the signal parameters estimation by the proposed PSO and the 4-parameters sine fitting algorithm.

\section{NUMERICAL TESTS}

The procedure is implemented in Matlab environment and numerical tests are executed to (i) verify the correctness of the procedure and its accuracy comparing the results with the 4parameters sine fitting algorithm that is the golden standard for this kind of analysis and (ii) determine the influence of sampling frequency and noise on the accuracy. The test signal considered is a sinusoidal waveform, with amplitude equal to $1 \mathrm{~V}$, frequency $49.7 \mathrm{~Hz}$, random phase and offset $0.1 \mathrm{~V}$. Moreover, in the test white Gaussian noise is superimposed on the signal. The Signal to noise ratio imposed is 50 $\mathrm{dB}$. The sampling frequency used to simulate the signal is equal to $1 \mathrm{kHz}$. The swarm is set to have a size of 20 particles and the inertia range is $[0.1$ $0.83]$

Fig. 5 shows the mean error of 2000 tests in the estimated parameters by the proposed method based on PSA and the 4-parameters sine fitting algorithm versus the number of samples processed. The number of samples considered are included in the range $[1.0,5.5] 10^{4}$ samples. The figure shows as the results for both the methods are comparable, in fact, the error has the same order of magnitude. Moreover, the test demonstrate as the estimation error decreases with the increasing of the number of samples that, by considering the execution time of Fig. 4 emphasize the advantage of the PSA method. In particular, for the sine fitting algorithm the amplitude estimation error decreases from $4.010^{-5} \mathrm{~V}$ to $0.810^{-5} \mathrm{~V}$. Instead, for the PSA the is included in the range $[4.0,0.7] 10^{-5} \mathrm{~V}$. The error estimation for the frequency, offset and phase is strongly influenced by the number of samples for both the method. The error for the frequency estimation decreases from $4.410^{-6} \mathrm{~Hz}$ to $0.410^{-6} \mathrm{~Hz}$ for the sine fitting, and from $4.910^{-6} \mathrm{~Hz}$ to $0.210^{-6} \mathrm{~Hz}$ for the PSA. The error in the offset estimation decreases from $2.510^{-5} \mathrm{~V}$ to $0.610^{-5} \mathrm{~V}$ and from $2.910^{-5} \mathrm{~V}$ to $0.410^{-5} \mathrm{~V}$. Finally, the phase estimation error decreases from $1.310^{-4} \mathrm{rad}$ to $0.310^{-4} \mathrm{rad}$ for the sine fitting and from $1.410^{-4} \mathrm{rad}$ to $0.210^{-4} \mathrm{rad}$ for the PSA.

In Fig. 6 is shown the standard deviation of the error in the frequency estimation versus the number of samples. The figure shows as the standard deviation for both the method is comparable and for a number of samples higher than $20 \mathrm{k}$ Samples the PSA shows an standard deviation lower than the sine fitting. The standard deviation of the error in the amplitude estimation is included in the range [2.3, $0.5] 10^{-5} \mathrm{~V}$ for the sine fitting and $[1.4,0.5] 10^{-5} \mathrm{~V}$ for the PSA. Instead, the standard deviation of the error in the phase estimation is included in the range $[2.8,1.0] 10^{-5} \mathrm{rad}$ for the sine fitting algorithm and $[2.5,0.9] 10^{-5} \mathrm{rad}$ for the PSA. Finally, the standard 
deviation of the error in the offset estimation is included in the range $[5.5,1.1] 10^{-6} \mathrm{~V}$ for the sine fitting and $[6.0,0.8] 10^{-56} \mathrm{~V}$ for the PSA.

Fig. 7 shows the mean error, for 200 tests, in the evaluation of the sinusoidal signal parameters versus the sampling frequency for the proposed method and the sine fitting algorithm. In the test, the number of samples analyzed is constant and equal to $10 \mathrm{k}$ samples and white Gaussian noise is superimposed to the signal with SNR equal to $50 \mathrm{~dB}$. Also in this case the error in the evaluation has the same order of magnitude for both the methods.
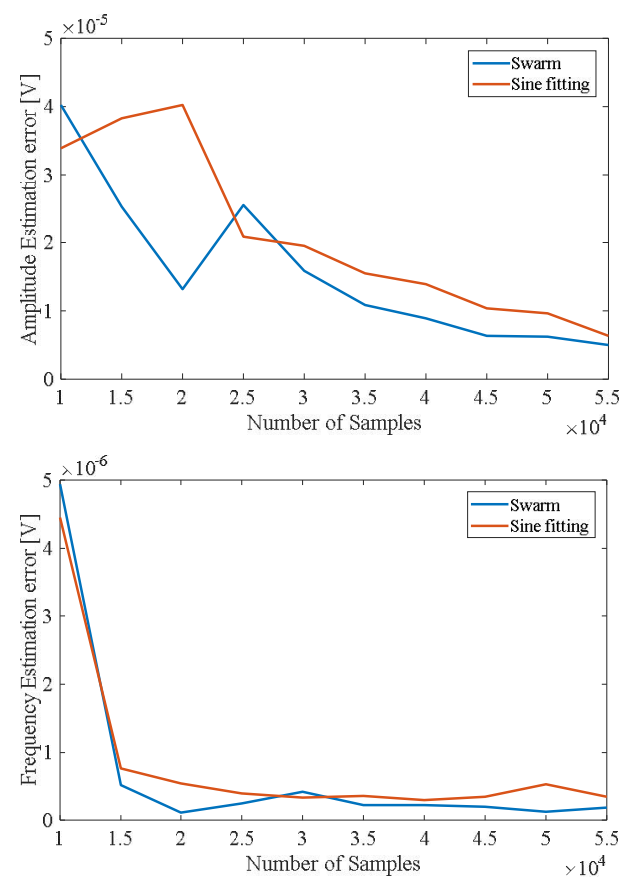

a)
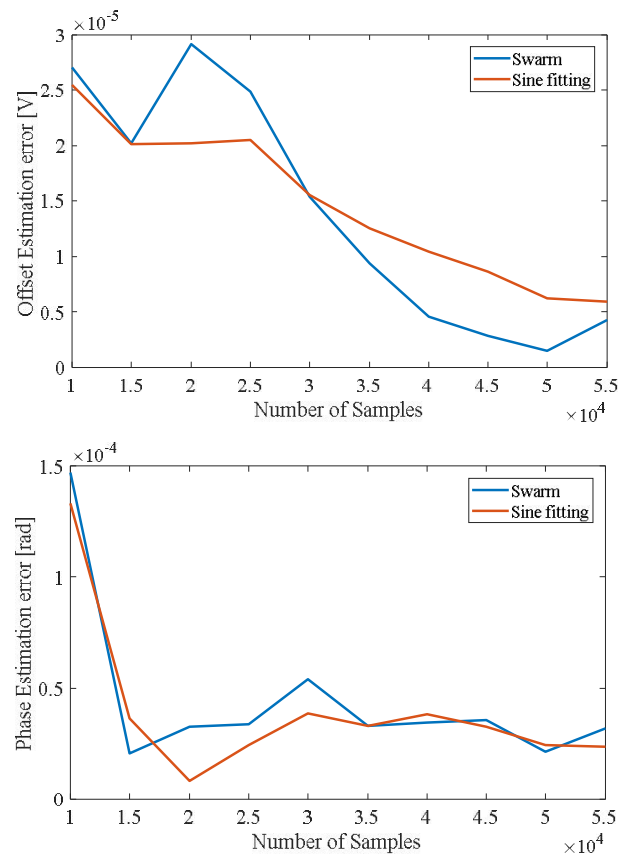

c)

d)

Fig. 5 - Mean error in the evaluation of a) amplitude, b) frequency, c) offset and d) phase for the proposed method and the 4-parameters sine fitting algorithm versus the number of samples analysed.

b)

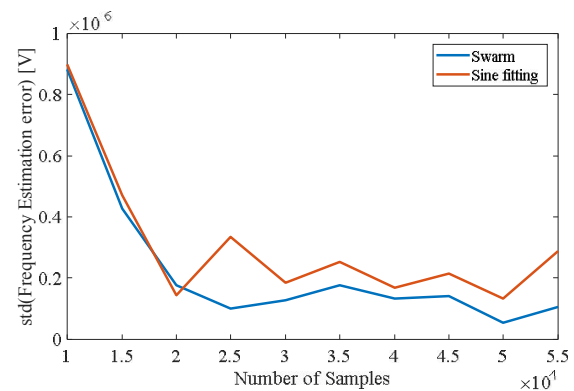

Fig. 6 - standard deviation of the error in the evaluation of the frequency for the proposed method and the 4-parameters sine fitting algorithm versus the number of samples analysed.
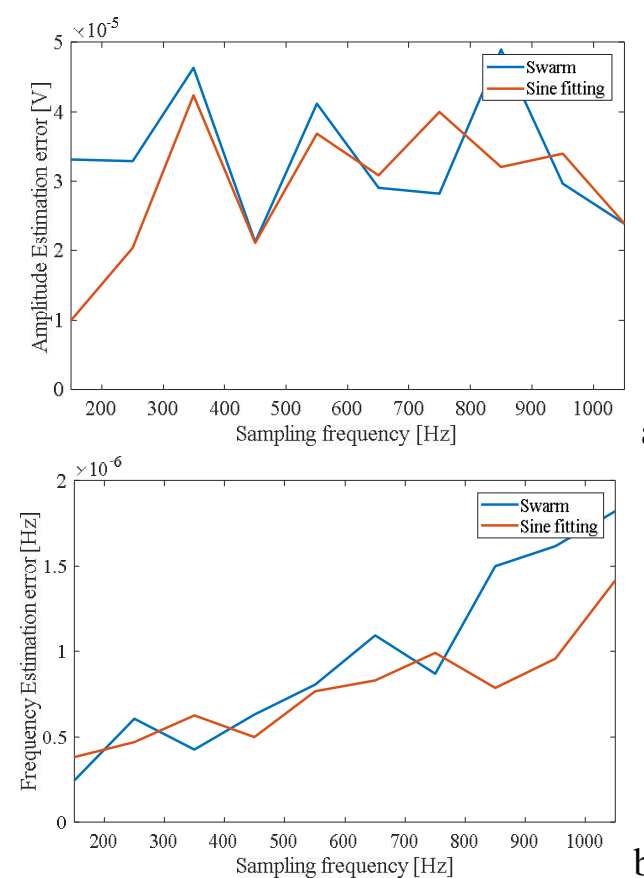

a)

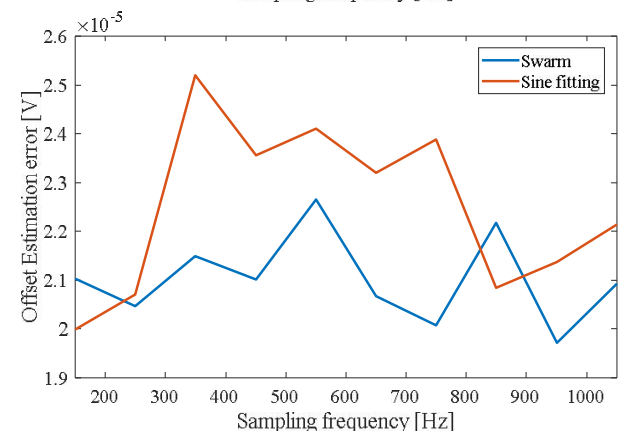

b)

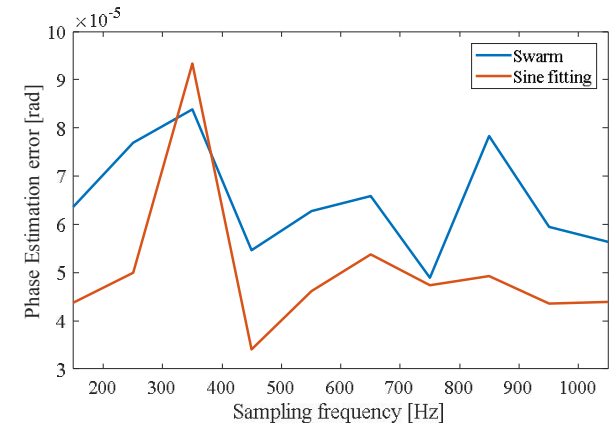

c)

Fig. 7 - Mean error in the evaluation of a) amplitude, b) frequency, c) offset and d) phase for the proposed method and the 4-parameters sine fitting algorithm versus the sampling frequency. 


\section{CONCLUSION}

In the paper, an innovative method for the sinewave signal parameters estimation is proposed. The method considers the parameters estimation as an optimization problem that is solved by using the Particle Swarm Algorithm.

Numerical tests show as the performance of the PSA are influenced by the objective function to be optimized by the algorithm. Moreover, the comparison of the test by considering the variation of number of samples processed and of the sampling frequency demonstrates as the parameters estimation error has the same order of magnitude of the sine fitting algorithm, that is the golden standard for this estimation.

The comparison of the execution for both the algorithm permits to assert that the Particle Swarm Algorithm can be considered an interesting technique for this problem solution in the case of reduced time response requirements.

\section{REFERENCES}

[1] T. S. Sidhu, "Accurate measurement of power system frequency using a digital signal processing technique," IEEE Trans. Instrum. Meas., vol. 48, no. 1, pp. 75-81, 1999.

[2] R. Aghazadeh, H. Lesani, M. Sanaye-Pasand, and B. Ganji, "New technique for frequency and amplitude estimation of power system signals," in IEE Proceedings: Generation, Transmission and Distribution, Vol. 152, No. 3, pp. 435-440, 2005.

[3] A. J. Roscoe, G. M. Burt, and J. R. McDonald, "Frequency and fundamental signal measurement algorithms for distributed control and protection applications," IET Gener. Transm. Distrib., Vol. 3, No. 5, pp. 485-495, 2009.

[4] D. L. Carnì and D. Grimaldi, "Comparative analysis of different acquisition techniques applied to static and dynamic characterization of high resolution DAC," in Proceedings of the 19th IMEKO World Congress 2009, 2009, Vol. 1, pp. 285-289.

[5] A. Baccigalupi, G. D'Alessandro, M. D'Arco, and R. S. L. Moriello, "Least square procedures to improve the results of the threeparameter sine-fitting algorithm," Acta IMEKO, Vol. 4, No. 2, pp. 100-106, 2015.

[6] F. C. Alegria, "Precision of the sinefittingbased total harmonic distortion estimator," Metrol. Meas. Syst., Vol. 23, No. 1, pp. 37-46, 2016.

[7] J. Šaliga, I. Kollár, L. Michaeli, J. Buša, J. Lipták, and T. Virosztek, "A comparison of least squares and maximum likelihood methods using sine fitting in ADC testing," Meas. J. Int. Meas. Confed., vol. 46, no. 10, pp. 4362-4368, 2013.

[8] Z.-N. Xu, F.-C. Lu, F.-C. Wang, and H.-M. Li, "Application of higher-order sine fitting algorithm with Hanning-windowed interpolated in dielectric loss angle measurement," Gaodianya Jishu/High Volt. Eng., Vol. 33, No. 4, pp. 50-53, 2007.

[9] D. L. Carní and G. Fedele, "Multi-Sine Fitting Algorithm enhancement for sinusoidal signal characterization," Comput. Stand. Interfaces, Vol. 34, No. 6, pp. 535-540, 2012.

[10] D. L. Carnì and G. Fedele, "Improved evaluation of initial condition for the multi-sine fitting algorithm," in Proc. of the 5th IEEE International Workshop on Intelligent Data Acquisition and Advanced Computing Systems: Technology and Applications, IDAACS, 2009, pp. 492-495.

[11] D. L. Carnì, "Static Characterization of AWG based on modified multi-sine fitting and zero crossing detection algorithms," J. Eng. Sci. Technol. Rev., vol. 10, no. 3, pp. 11-17, 2017.

[12] D. L. Carnì and D. Grimaldi, "Characterization of high resolution DAC by DFT and sine fitting," in Proc. of IEEE Intrumentation and Measurement Technology Conference, 2009, pp. 1250-1253.

[13] F. De Rango, M. Tropea, A. F. Santamaria, and S. Marano, "An enhanced QoS CBT multicast routing protocol based on Genetic Algorithm in a hybrid HAP-Satellite system," Comput. Commun., vol. 30, no. 16, pp. 3126-3143, 2007.

[14] F. De Rango and M. Tropea, "Energy saving and load balancing in wireless ad hoc networks through ant-based routing," in International Symposium on Performance Evaluation of Computer and Telecommunication Systems 2009, SPECTS 2009, Part of the 2009 Summer Simulation Multiconference, SummerSim 2009, 2009.

[15] A. Atyabi and S. Samadzadegan, Particle swarm optimization: A survey. 2011.

[16] X. Hu, R. C. Eberhart, and Y. Shi, "Engineering Optimization with Particle Swarm," in Swarm Intelligence Symposium 2003, 2003.

[17] R. Poli, J. Kennedy, and T. Blackwell, "Particle swarm optimization: An overview," Swarm Intell., 2007.

[18] P. Eberhard and Q. Tang, "Particle swarm optimization used for mechanism design and guidance of swarm mobile robots," in Particle Swarm Optimization: Theory, Techniques and Applications, 2011. 
[19] N. Forghani, M. Forouzanfar, and A. Eftekhari, "Application of Particle Swarm Optimization in Accurate Segmentation of Brain MR Images," Part. Swarm Optim., 2008.

[20] M. Clerc, Particle Swarm Optimization. 2006.

[21] S. Kessentini and D. Barchiesi, "Convergence criteria for the particle swarm optimization in a full iterative process," in 2017 IEEE Congress on Evolutionary Computation, CEC 2017 Proceedings, 2017.

[22] P. M. Ramos and A. C. Serra, "A new sinefitting algorithm for accurate amplitude and phase measurements in two channel acquisition systems," Meas. J. Int. Meas. Confed., Vol. 41, No. 2, pp. 135-143, 2008.

[23] T. Z. Bilau, T. Megyeri, A. Sárhegyi, J. Márkus, and I. Kollár, "Four-parameter fitting of sine wave testing result: Iteration and convergence," Comput. Stand. Interfaces,
Vol. 26, No. 1, pp. 51-56, 2004.

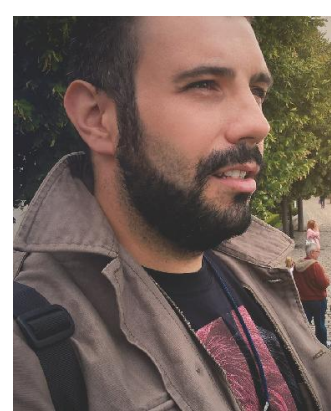

Pierfrancesco Raimondo, achieved a master's degree in computer science engineering in March 2011. Since 2012 he has been a research fellow at University of Calabria. Currently he is a PhD student in Information and Communication Technology at the University of Calabria.

His research concerns data transmission in adhoc networks, optimization and simulation algorithms and models, IoT, Vanet, simulation and modeling, embedded systems, heuristic algorithms. 